\section{Sampled-Data Synchronization Control of Dynamical Networks with Stochastic Sampling}

\author{
Bo Shen, Zidong Wang and Xiaohui Liu
}

\begin{abstract}
This paper is concerned with the sampled-data synchronization control problem for a class of dynamical networks. The sampling period considered here is assumed to be timevarying that switches between two different values in a random way with given probability. The addressed synchronization control problem is first formulated as an exponentially mean-square stabilization problem for a new class of dynamical networks that involve both the multiple probabilistic interval delays (MPIDs) and the sector-bounded nonlinearities (SBNs). Then, a novel Lyapunov functional is constructed to obtain sufficient conditions under which the dynamical network is exponentially mean-square stable. Both Gronwall's inequality and Jenson integral inequality are utilized to substantially simplify the derivation of the main results. Subsequently, a set of sampled-data synchronization controllers is designed in terms of the solution to certain matrix inequalities that can be solved effectively by using available software. Finally, a numerical simulation example is employed to show the effectiveness of the proposed sampled-data synchronization control scheme.
\end{abstract}

Keywords - Dynamical networks; Synchronization control; Sampled-data control; Stochastic sampling; Gronwall's inequality; Jenson integral inequality.

\section{INTRODUCTION}

Complex networks have recently received much attention due to their extensive applications in both science and engineering such as Internet, World Wide Web, food webs, electric power grids, cellular and metabolic networks, scientific citation networks, social networks, etc. In the real world, there exist a number of classes of networks in which the state of each node evolves asynchronously. Therefore, for such asynchronous networks, it is of great significance to investigate whether there exist controllers that can synchronize all the nodes and, if such controllers exist, how to actually design them. The synchronization problem has recently been paid a great deal of efforts and some effective synchronization control schemes have been proposed for a variety of non-synchronous networks $[1,6,15,17,18,20,21,23,25]$.

With the rapid development of high-speed computers, modern control systems tend to be controlled by digital controllers, i.e., only the samples of the control input signals at discrete time instants will be employed. A crucial issue arose here is that the variation of sampling periods may drastically deteriorate the desired performance of controlled systems under investigation. As such, it is of great importance to investigate 1) how the considered system is affected by the controller based on sampled-data; and 2) how to design a sampled-data controller to guarantee the desired performance of controlled system. In fact, the sampleddata control problem has been a research focus for nearly three

\footnotetext{
This work was supported in part by the Engineering and Physical Science Research Council (EPSRC) of the UK under Grant GR/S27658/01, the Roya Society of the UK, the National Natural Science Foundation of China under Grants 61028008, 60974030, 61134009 and 61104125, the National 973 Program of China under Grant 2009CB320600, and the Alexander von Humboldt Foundation of Germany.

B. Shen is with the School of Information Science and Technology, Donghua University, Shanghai 200051, China. Email: shenbodh@gmail.com

Z. Wang is with the Department of Information Systems and Computing, Brunel University, Uxbridge, Middlesex, UB8 3PH, United Kingdom. He is also with the School of Information Science and Technology, Donghua University, Shanghai 200051, China. Email: Zidong. Wang@brunel.ac.uk

$\mathrm{X}$. Liu is with the Department of Information Systems and Computing, Brunel University, Uxbridge, Middlesex, UB8 3PH, United Kingdom. Email: Xiaohui.Liu@brunel.ac.uk
}

decades and numerous results have been reported in the literature $[2,5,22]$. Recently, with the ever developing techniques for tackling time delays that frequently occur in various engineering systems (see e.g. $[3,8,10,12-14]$ ), an arguably popular approach to dealing with sampled-data control problems, which has been proposed in [8], is to transform the sampling period into a certain time-delay with finite bound. By using such an approach, the sampled-data analysis problem amounts to the corresponding problem for a time delayed system. In this regard, the sampled-data $H_{\infty}$ control problem has been thoroughly investigated in [9] for sampled-data systems with stochastic sampling periods.

Apparently, in nowadays digitalized world, it is of both theoretical significance and practical importance to analyze how a digitalized control signal would influence the synchronization behavior of a continuous-time dynamical network. In other words, there is a vital need to investigate the sampled-data synchronization for dynamical networks. Unfortunately, although sampled-data control technologies have been developed relatively well in control theory, the particular sampled-data synchronization problem for dynamical networks has so far received very little attention due mainly to the mathematical complexity. Indeed, the essential difficulties would be 1) how to handle the dynamical network itself in terms of the couplings, nonlinearities and external disturbances; 2) how to deal with the stochastic sampling by quantifying the its impact on the synchronization in terms of the sampling occurrence probability; and 3) how to actually design a set of easy-to-implement sampled-data controllers in order to synchronize the dynamical network especially when the original dynamical network is unstable. It is, therefore, the main aim of this paper to challenge the sampled-data synchronization problem for dynamical networks by overcoming the aforementioned three major difficulties.

In this paper, the sampled-data synchronization control problem is addressed for a class of dynamical networks. The sampling period considered here is time-varying that is allowed to switch between two different values in a random way. Note that the stochastic sampling problem typically occurs in a networked environment where the signal sampling is subject to unpredictable perturbations. Following the idea proposed in $[9,24]$, the addressed synchronization control problem is formulated as a problem of stability analysis for a dynamical network with multiple probabilistic interval delays (MPIDs) as well as sector-bounded nonlinearities (SBNs). It is worth mentioning that the MPIDs in the transformed dynamical model are quite general which have not been addressed before. By utilizing both the Gronwall's inequality and the Jenson integral inequality, sufficient conditions are derived under which the dynamical network is exponentially mean-square stable. A numerical simulation example is exploited to demonstrate the effectiveness of the proposed synchronization control scheme.

\section{Problem Formulation and Preliminaries}

Consider the following dynamical network consisting of $N$ coupled nodes of the form:

$$
\dot{x}_{i}(t)=f\left(x_{i}(t)\right)+\sum_{j=1}^{N} w_{i j} \Gamma x_{j}(t)+u_{i}(t)
$$

for all $i=1,2, \cdots, N$, where $x_{i}(t) \in \mathbb{R}^{n}$ is the state vector of the $i$ th node and $u_{i}(t) \in \mathbb{R}^{n}$ is the control input of the $i$ th node. $\Gamma=$ $\operatorname{diag}\left\{r_{1}, r_{2}, \cdots, r_{n}\right\}$ is a matrix linking the $j$ th state variable if $r_{j} \neq 0$, and $W=\left(w_{i j}\right)_{N \times N}$ is the coupled configuration matrix of the network with $w_{i j} \geq 0(i \neq j)$ but not all zero. As usual, 
the coupling configuration matrix $W=\left(w_{i j}\right)_{N \times N}$ is symmetric (i.e., $\left.W=W^{T}\right)$ and satisfies $\sum_{j=1}^{N} w_{i j}=\sum_{j=1}^{N} w_{j i}=0$ for all $i=1,2, \cdots, N$.

The nonlinear vector-valued function $f: \mathbb{R}^{n} \rightarrow \mathbb{R}^{n}$ is assumed to be continuous and satisfy the following sector-bounded condition [16]:

$$
\left[f(x)-f(y)-U_{1}(x-y)\right]^{T}\left[f(x)-f(y)-U_{2}(x-y)\right] \leq 0
$$

for all $x, y \in \mathbb{R}^{n}$, where $U_{1}$ and $U_{2}$ are constant matrices of appropriate dimensions. Note that the sector-like description of the nonlinearities in (2) is more general than the usual Lipschitz functions. By adopting such a presentation, it would be possible to reduce the conservatism of the main results caused by quantifying the nonlinear functions via a matrix inequality technique.

Denote by $s(t) \in \mathbb{R}^{n}$ the solution to the unforced isolate node $\dot{s}(t)=f(s(t))$ and let the error vector be $e_{i}(t)=x_{i}(t)-s(t)$. Then, the error dynamics of network (1) can be easily obtained as follows:

$$
\dot{e}_{i}(t)=\tilde{f}\left(x_{i}(t), s(t)\right)+\sum_{j=1}^{N} w_{i j} \Gamma e_{j}(t)+u_{i}(t)
$$

for all $i=1,2, \cdots, N$, where $\tilde{f}\left(x_{i}(t), s(t)\right)=f\left(x_{i}(t)\right)-f(s(t))$.

In this paper, the control input $u_{i}(t)$ is sampled before entering the network (3), which gives rise to the sampled-data analysis problem. More specifically, for every $i(1 \leq i \leq N)$, the control signal is generated by a zero-order hold function with a sequence of hold times $0=t_{0}^{i}<t_{1}^{i}<\cdots<t_{k}^{i}<\cdots$

$$
u_{i}(t)=u_{i d}\left(t_{k}^{i}\right)=K e_{i}\left(t_{k}^{i}\right), \quad t_{k}^{i} \leq t<t_{k+1}^{i}
$$

where $u_{i d}(\cdot)$ is a discrete-time control signal, $t_{k}^{i}$ denotes the sampling instant of $i$ th node and satisfies $\lim _{k \rightarrow \infty} t_{k}^{i}=\infty$, and $K$ is the feedback gain to be determined.

Noting that $t_{k}^{i}=t-\left(t-t_{k}^{i}\right):=t-d_{k}^{i}(t)$ holds for $t_{k}^{i} \leq t<t_{k+1}^{i}$, the closed-loop error dynamics of the network is governed by

$$
\dot{e}_{i}(t)=\tilde{f}\left(x_{i}(t), s(t)\right)+\sum_{j=1}^{N} w_{i j} \Gamma e_{j}(t)+K e_{i}\left(t-d_{k}^{i}(t)\right),
$$

$t_{k}^{i} \leq t<t_{k+1}^{i}$, for all $i=1,2, \cdots, N$.

By defining a function $d_{i}(t)$ in the whole period of time $[0, \infty)$ as follows:

$$
d_{i}(t)=d_{k}^{i}(t), \quad t_{k}^{i} \leq t<t_{k+1}^{i}, \quad k=0,1,2, \cdots, \infty,
$$

the closed-loop error dynamics of the network defined on $[0, \infty)$ can be derived immediately as follows:

$$
\dot{e}_{i}(t)=\tilde{f}\left(x_{i}(t), s(t)\right)+\sum_{j=1}^{N} w_{i j} \Gamma e_{j}(t)+K e_{i}\left(t-d_{i}(t)\right)
$$

where $i=1,2, \cdots, N$.

As discussed in the introduction, in a networked environment, the sampling period itself might be a stochastic variable due to unpredictable environmental changes. To reflect such a reality, in this paper, the sampling period of each control signal is allowed to randomly switch between two different values $p_{1}$ and $p_{2}$ with $0<p_{1}<p_{2}$. Such a phenomena is referred to as stochastic sampling [9], and can be represented by utilizing a set of random variables $\varrho_{i}(1 \leq i \leq N)$ with probabilities $\operatorname{Prob}\left\{\varrho_{i}=p_{1}\right\}=\beta_{i}$ and $\operatorname{Prob}\left\{\varrho_{i}=p_{2}\right\}=1-\beta_{i}$ where $\beta_{i} \in[0,1]$ is a known constant.
Remark 1: In the control community, various sampling issues have been raised and thoroughly studied, e.g. single-rate sampling, multi-rate sampling and time-varying sampling. Note that these sampling methods are customarily assumed to be implemented in a deterministic way. However, in practical engineering within a networked environment, the sampling process itself might be subject to random abrupt changes, for example, sudden environment changes, random sampler failures, etc. In other words, the sampling periods may vary in a probabilistic way. Such a phenomena of sampling process, namely, stochastic sampling, has been largely overlooked in the area of coupled dynamical networks. In this paper, we are only concerned with the special case that the sampling period is allowed to randomly switch between two different values. Our results obtained later can be easily extended to more complex cases where there are multiple sampling rates. Note that the stochastic sampling problem has been addressed in [9] for sampled-data control problem and in [7] for computer graphics problem.

From the definition of $d_{i}(t)$, it is obvious that $d_{i}(t)$ is a sawtooth function with randomness, and its value lies in the interval $\left[0, p_{2}\right]$. We take the interval $\left[0, p_{2}\right]$ apart into two interval $\left[0, p_{1}\right]$ and $\left(p_{1}, p_{2}\right]$, and introduce a new random variable $\gamma_{i}(t)$ :

$$
\gamma_{i}(t)=\left\{\begin{array}{cc}
1, & 0 \leq d_{i}(t)<p_{1} \\
0, & p_{1} \leq d_{i}(t) \leq p_{2}
\end{array}\right.
$$

According to [9], we have $\operatorname{Prob}\left\{\gamma_{i}(t)=1\right\}=\alpha_{i}$ and $\operatorname{Prob}\left\{\gamma_{i}(t)=0\right\}=1-\alpha_{i}$ where $\alpha_{i}=\beta_{i}+\frac{p_{1}}{p_{2}}\left(1-\beta_{i}\right)$.

Following the idea proposed in [9,24], for every $i(1 \leq i \leq N)$, we define functions $\tau_{1}^{i}: \mathbb{R} \rightarrow\left[0, p_{1}\right]$ and $\tau_{2}^{i}: \mathbb{R} \rightarrow\left[p_{1}, p_{2}\right]$ such that

$$
\tau_{1}^{i}(t)=\left\{\begin{array}{cc}
d_{i}(t), & \gamma_{i}(t)=1, \\
p_{1}, & \gamma_{i}(t)=0,
\end{array} \quad \tau_{2}^{i}(t)=\left\{\begin{array}{cc}
p_{1}, & \gamma_{i}(t)=1 \\
d_{i}(t), & \gamma_{i}(t)=0
\end{array}\right.\right.
$$

and rewrite the system (6) equivalently as

$$
\begin{aligned}
\dot{e}_{i}(t)= & \tilde{f}\left(x_{i}(t), s(t)\right)+\sum_{j=1}^{N} w_{i j} \Gamma e_{j}(t)+\gamma_{i}(t) K e_{i}\left(t-\tau_{1}^{i}(t)\right) \\
& +\left(1-\gamma_{i}(t)\right) K e_{i}\left(t-\tau_{2}^{i}(t)\right)
\end{aligned}
$$

for all $i=1,2, \cdots, N$.

Furthermore, the coupled systems (7) can be converted into the following compact form:

$$
\begin{aligned}
\dot{e}(t)= & (W \otimes \Gamma) e(t)+g(x(t), s(t))+\sum_{i=1}^{N} \gamma_{i} C_{i} e\left(t-\tau_{1}^{i}(t)\right) \\
& +\sum_{i=1}^{N}\left(1-\gamma_{i}\right) C_{i} e\left(t-\tau_{2}^{i}(t)\right)
\end{aligned}
$$

where

$$
\begin{aligned}
& e(t)=\left[\begin{array}{llll}
e_{1}^{T}(t) & \cdots & e_{N}^{T}(t)
\end{array}\right]^{T}, x(t)=\left[\begin{array}{lll}
x_{1}^{T}(t) & \cdots & x_{N}^{T}(t)
\end{array}\right]^{T}, \\
& g(x(t), s(t))=\left[\begin{array}{lll}
\tilde{f}^{T}\left(x_{1}(t), s(t)\right) & \cdots & \tilde{f}^{T}\left(x_{N}(t), s(t)\right)
\end{array}\right]^{T}, \\
& C_{i}=\operatorname{diag}\{\underbrace{0, \cdots, 0}_{i-1}, K, 0, \cdots, 0\} .
\end{aligned}
$$

Next, it follows from (2) that the nonlinear function $g(x(t), s(t))$ satisfies

$$
\left[\begin{array}{c}
e(t) \\
g(x(t), s(t))
\end{array}\right]^{T}\left[\begin{array}{cc}
\tilde{U}_{1 \Lambda} & \tilde{U}_{2 \Lambda} \\
* & I
\end{array}\right]\left[\begin{array}{c}
e(t) \\
g(x(t), s(t))
\end{array}\right] \leq 0
$$


where

$$
\begin{aligned}
& \tilde{U}_{1 \Lambda}=\frac{U_{1 \Lambda}^{T} U_{2 \Lambda}+U_{2 \Lambda}^{T} U_{1 \Lambda}}{2}, \quad \tilde{U}_{2 \Lambda}=-\frac{U_{1 \Lambda}^{T}+U_{2 \Lambda}^{T}}{2}, \\
& U_{1 \Lambda}=\operatorname{diag}_{N}\left\{U_{1}\right\}, \quad U_{2 \Lambda}=\operatorname{diag}_{N}\left\{U_{2}\right\} .
\end{aligned}
$$

The following definitions are needed for stating the problem to be investigated.

Definition 1: The system (8) is said to be exponentially mean-square stable if there exist two constants $\nu>0$ and $\delta>0$ such that

$$
\mathbb{E}\left\{\|e(t)\|^{2}\right\} \leq \nu e^{-\delta t} \sup _{-2 p_{2} \leq \theta \leq 0} \mathbb{E}\left\{\|\phi(\theta)\|^{2}\right\}
$$

where $\phi(\cdot)$ is the initial function of system (8) defined as $e(t)=$ $\phi(t), t \in\left[-2 p_{2}, 0\right]$.

Definition 2: The dynamical network (1) is said to be exponentially mean-square synchronized if the system (8) is exponentially mean-square stable.

We are now ready to state the problem to be investigated. In this paper, we aim to design a set of controllers of the form (4) to achieve the exponential mean-square synchronization for the dynamical network (1). In other words, we are interested in looking for a gain matrix $K$ such that the system (8) is exponentially mean-square stable.

\section{MAin Results}

Let us start with stability analysis for the augmented system (8). For the sake of simplicity, we denote

$$
\begin{aligned}
\mathcal{F}(t):= & (W \otimes \Gamma) e(t)+g(x(t), s(t))+\sum_{i=1}^{N} \alpha_{i} C_{i} e\left(t-\tau_{1}^{i}(t)\right) \\
& +\sum_{i=1}^{N}\left(1-\alpha_{i}\right) C_{i} e\left(t-\tau_{2}^{i}(t)\right), \\
\mathcal{G}_{i}(t):= & \sqrt{\alpha_{i}\left(1-\alpha_{i}\right)} C_{i}\left(e\left(t-\tau_{1}^{i}(t)\right)-e\left(t-\tau_{2}^{i}(t)\right)\right) .
\end{aligned}
$$

Construct a Lyapunov functional as follows:

$$
V\left(e_{t}\right)=V_{1}\left(e_{t}\right)+V_{2}\left(e_{t}\right)+V_{3}\left(e_{t}\right)
$$

where

$$
\begin{aligned}
& V_{1}\left(e_{t}\right)=e^{T}(t) \operatorname{Pe}(t), \\
& V_{2}\left(e_{t}\right)=\int_{t-p_{1}}^{t} e^{T}(s) Q_{1} e(s) d s+\int_{t-p_{2}}^{t-p_{1}} e^{T}(s) Q_{2} e(s) d s, \\
& V_{3}\left(e_{t}\right)=\sum_{i=1}^{N} \int_{-p_{1}}^{0} \int_{t+\omega}^{t}\left(\mathcal{F}^{T}(s) Z_{1}^{i} \mathcal{F}(s)+\sum_{j=1}^{N} \mathcal{G}_{j}^{T}(s) Z_{1}^{i} \mathcal{G}_{j}(s)\right) d s d \omega \\
&+\sum_{i=1}^{N} \int_{-p_{2}}^{-p_{1}} \int_{t+\omega}^{t}\left(\mathcal{F}^{T}(s) Z_{2}^{i} \mathcal{F}(s)+\sum_{j=1}^{N} \mathcal{G}_{j}^{T}(s) Z_{2}^{i} \mathcal{G}_{j}(s)\right) d s d \omega,
\end{aligned}
$$

in which $Q_{1}>0, Q_{2}>0, Z_{1}^{i}>0 Z_{2}^{i}>0(1 \leq i \leq N)$, and $P>0$ are positive definite matrices to be determined.

Define the infinitesimal operator $\mathcal{L}$ of $V\left(e_{t}\right)$ as follows:

$$
\mathcal{L} V\left(e_{t}\right)=\lim _{\Delta \rightarrow 0^{+}} \frac{1}{\Delta}\left\{\mathbb{E}\left\{V\left(e_{t+\Delta}\right) \mid e_{t}\right\}-V\left(e_{t}\right)\right\}
$$

The following lemma provides a sufficient condition to guarantee the exponential mean-square stability of the system (8).

Lemma 1: Let the matrices $P>0, Q_{1}>0, Q_{2}>0, Z_{1}^{i}>0$, $Z_{2}^{i}>0(1 \leq i \leq N)$ and $K$ be given. For the Lyapunov functional defined in (10), if there exists a scalar $\varepsilon>0$ such that

$$
\mathbb{E}\left\{\mathcal{L} V\left(e_{t}\right)\right\}<-\varepsilon \mathbb{E}\left\{\|e(t)\|^{2}\right\}
$$

then the augmented system (8) is exponentially means-square stable.

Proof: From the definition of Lyapunov functional (10), we have

$$
\begin{aligned}
& V\left(e_{t}\right) \\
\leq & \lambda_{\max }\left(Q_{1}\right) \int_{t-p_{1}}^{t}\|e(s)\|^{2} d s+\lambda_{\max }\left(Q_{2}\right) \int_{t-p_{2}}^{t-p_{1}}\|e(s)\|^{2} d s \\
& +\lambda_{\max }(P)\|e(t)\|^{2}+\sum_{i=1}^{N}\left(p_{1} \lambda_{\max }\left(Z_{1}^{i}\right)+\left(p_{2}-p_{1}\right) \lambda_{\max }\left(Z_{2}^{i}\right)\right) \\
& \times \int_{t-p_{2}}^{t}\left(\|\mathcal{F}(s)\|^{2}+\sum_{j=1}^{N}\left\|\mathcal{G}_{j}(s)\right\|^{2}\right) d s .
\end{aligned}
$$

In order to estimate the upper bound of the nonlinear function $g(x(t), s(t))$, we rewrite it as

$$
g(x(t), s(t))=\frac{U_{1 \Lambda}+U_{2 \Lambda}}{2} e(t)+\tilde{g}(x(t), s(t))
$$

where $\tilde{g}(x(t), s(t))$ satisfies $\|\tilde{g}(x(t), s(t))\|^{2} \leq\left\|\frac{U_{1 \Lambda}-U_{2 \Lambda}}{2} e(t)\right\|^{2}$.

Denoting $c_{1}=\frac{1}{2}\left(\left\|U_{1 \Lambda}+U_{2 \Lambda}\right\|^{2}+\left\|U_{1 \Lambda}-U_{2 \Lambda}\right\|^{2}\right)$, we have $\|g(x(t), s(t))\|^{2} \leq c_{1}\|e(t)\|^{2}$, and therefore it follows that

$\int_{t-p_{2}}^{t}\left(\|\mathcal{F}(s)\|^{2}+\sum_{j=1}^{N}\left\|\mathcal{G}_{j}(s)\right\|^{2}\right) d s \leq c_{2} \sup _{t-2 p_{2} \leq \theta \leq t}\left\{\|e(\theta)\|^{2}\right\}$

where

$$
\begin{aligned}
c_{2}= & 4 p_{2}\left(\|W \otimes \Gamma\|^{2}+c_{1}+N \sum_{i=1}^{N}\left\|\alpha_{i} C_{i}\right\|^{2}\right. \\
& \left.+N \sum_{i=1}^{N}\left\|\left(1-\alpha_{i}\right) C_{i}\right\|^{2}+\sum_{i=1}^{N}\left\|\sqrt{\alpha_{i}\left(1-\alpha_{i}\right)} C_{i}\right\|^{2}\right) .
\end{aligned}
$$

Substituting (14) into (13) yields

$$
a_{1}\|e(t)\|^{2} \leq V\left(e_{t}\right) \leq a_{2}\left(2+p_{2}\right) \sup _{t-2 p_{2} \leq \theta \leq t}\left\{\|e(\theta)\|^{2}\right\}
$$

where

$$
\begin{aligned}
a_{1}= & \lambda_{\min }(P), \\
a_{2}= & \max \left\{\lambda_{\max }(P), \lambda_{\max }\left(Q_{1}\right), \lambda_{\max }\left(Q_{2}\right),\right. \\
& \left.c_{2} \sum_{i=1}^{N}\left(p_{1} \lambda_{\max }\left(Z_{1}^{i}\right)+\left(p_{2}-p_{1}\right) \lambda_{\max }\left(Z_{2}^{i}\right)\right)\right\} .
\end{aligned}
$$

Noticing (12) and (15), we have

$$
\begin{aligned}
& \mathbb{E}\left\{V\left(e_{t}\right)\right\}=\mathbb{E}\left\{V\left(e_{0}\right)\right\}+\mathbb{E}\left\{\int_{0}^{t} \mathcal{L} V\left(e_{s}\right) d s\right\} \\
& \leq a_{2}\left(2+p_{2}\right) \sup _{-2 p_{2} \leq \theta \leq 0} \mathbb{E}\left\{\|\phi(\theta)\|^{2}\right\}-\int_{0}^{t} \varepsilon \mathbb{E}\left\{\|e(s)\|^{2}\right\} d s .
\end{aligned}
$$

Moreover, by considering $V\left(e_{t}\right) \geq a_{1}\|e(t)\|^{2}$, we obtain

$$
\begin{aligned}
\mathbb{E}\left\{\|e(t)\|^{2}\right\} \leq & \frac{a_{2}\left(2+p_{2}\right)}{a_{1}} \sup _{-2 p_{2} \leq \theta \leq 0} \mathbb{E}\left\{\|\phi(\theta)\|^{2}\right\} \\
& -\int_{0}^{t} \frac{\varepsilon}{a_{1}} \mathbb{E}\left\{\|e(s)\|^{2}\right\} d s .
\end{aligned}
$$


Finally, it follows from the Gronwall's inequality [19] that

$$
\mathbb{E}\left\{\|e(t)\|^{2}\right\} \leq \frac{a_{2}\left(2+p_{2}\right)}{a_{1}} e^{-\frac{\varepsilon}{a_{1}} t} \sup _{-2 p_{2} \leq \theta \leq 0} \mathbb{E}\left\{\|\phi(\theta)\|^{2}\right\}
$$

which, from Definition 1, means that the augmented system (8) is exponentially mean-square stable. The proof is complete.

Remark 2: Note that, in the proof of Lemma 1, the Gronwall's inequality is employed to prove the exponential mean-square stability for a time-delay system under the condition (12). Such an approach is different from the conventional ones in the literature, which simplifies the procedure for proving the exponential mean-square stability.

The following lemma will be used in deriving our main results.

Lemma 2: [11] For any constant matrix $M \in \mathbb{R}^{m \times m}, M=$ $M^{T}>0$, scalar $p>0$, vector function $x:[0, p] \rightarrow \mathbb{R}^{m}$ such that the integration in the sequel is well defined, the following holds

$$
p \int_{0}^{p} x^{T}(s) M x(s) d s \geq\left(\int_{0}^{p} x(s) d s\right)^{T} M\left(\int_{0}^{p} x(s) d s\right) .
$$

By resorting to Lemma 1 and Lemma 2, we present a stability condition for the augmented system (8) in the following theorem.

Theorem 1: For a given a controller gain matrix $K$, the augmented system (8) is exponentially mean-square stable if there exist matrices $P>0, Q_{1}>0, Q_{2}>0, Z_{1}^{i}>0, Z_{2}^{i}>0$ $(1 \leq i \leq N)$ and a scalar $\lambda>0$ satisfying

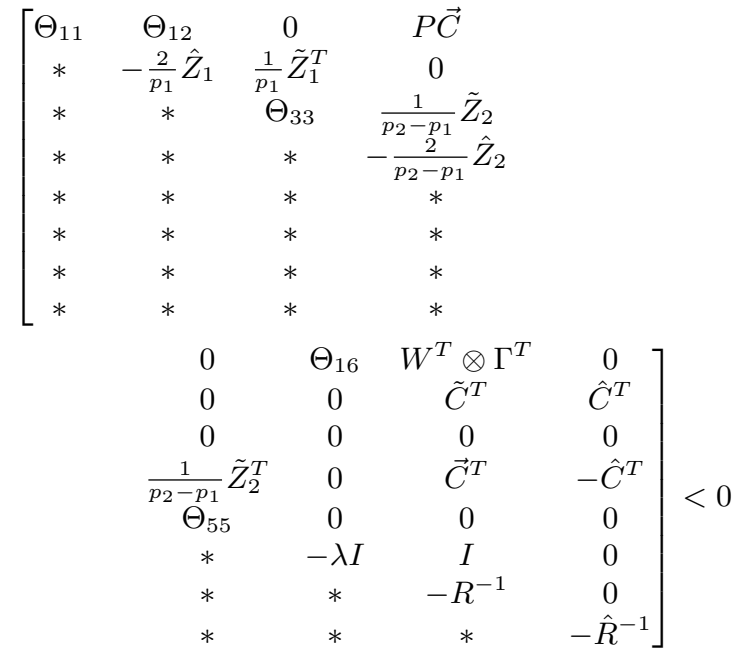

where

$$
\begin{aligned}
& \Theta_{11}=P(W \otimes \Gamma)+(W \otimes \Gamma)^{T} P+Q_{1}-\frac{1}{p_{1}} \sum_{i=1}^{N} Z_{1}^{i}-\lambda \tilde{U}_{1 \Lambda}, \\
& \Theta_{12}=P \tilde{C}+\frac{1}{p_{1}} \tilde{Z}_{1}, \quad \Theta_{16}=P-\lambda \tilde{U}_{2 \Lambda}, \\
& \Theta_{33}=-Q_{1}+Q_{2}-\frac{1}{p_{1}} \sum_{i=1}^{N} Z_{1}^{i}-\frac{1}{p_{2}-p_{1}} \sum_{i=1}^{N} Z_{2}^{i}, \\
& \Theta_{55}=-Q_{2}-\frac{1}{p_{2}-p_{1}} \sum_{i=1}^{N} Z_{2}^{i}, R=\sum_{i=1}^{N}\left(p_{1} Z_{1}^{i}+\left(p_{2}-p_{1}\right) Z_{2}^{i}\right), \\
& \hat{R}=\operatorname{diag}_{N}\left\{\begin{array}{ll}
R\}, & \tilde{C}=\left[\alpha_{1} C_{1} \quad \cdots \quad \alpha_{N} C_{N}\right.
\end{array}\right] \\
& \vec{C}=\left[\begin{array}{lll}
\left(1-\alpha_{1}\right) C_{1} \quad \cdots \quad\left(1-\alpha_{N}\right) C_{N}
\end{array}\right], \\
& \hat{C}=\operatorname{diag}\left\{\sqrt{\alpha_{1}\left(1-\alpha_{1}\right)} C_{1}, \cdots, \sqrt{\alpha_{N}\left(1-\alpha_{N}\right)} C_{N}\right\} \\
& \tilde{Z}_{1}=\left[\begin{array}{lll}
Z_{1}^{1} & \cdots & Z_{1}^{N}
\end{array}\right], \quad \hat{Z}_{1}=\operatorname{diag}\left\{Z_{1}^{1}, \cdots, Z_{1}^{N}\right\} \\
& \tilde{Z}_{2}=\left[\begin{array}{lll}
Z_{2}^{1} & \cdots & Z_{2}^{N}
\end{array}\right], \quad \hat{Z}_{2}=\operatorname{diag}\left\{Z_{2}^{1}, \cdots, Z_{2}^{N}\right\} .
\end{aligned}
$$

Proof: Consider the Lyapunov functional defined in (10) and calculate $\mathbb{E}\left\{\mathcal{L} V\left(e_{t}\right)\right\}$ along the dynamics of system (8) as follows:

$$
\begin{aligned}
\mathbb{E} & \left\{\mathcal{L} V\left(e_{t}\right)\right\} \\
= & \mathbb{E}\left\{e^{T}(t) Q_{1} e(t)-e^{T}\left(t-p_{1}\right)\left(Q_{1}-Q_{2}\right) e\left(t-p_{1}\right)\right. \\
& -e^{T}\left(t-p_{2}\right) Q_{2} e\left(t-p_{2}\right)+2 e^{T}(t) P \mathcal{F}(t) \\
& +\mathcal{F}^{T}(t) \sum_{i=1}^{N}\left(p_{1} Z_{1}^{i}+\left(p_{2}-p_{1}\right) Z_{2}^{i}\right) \mathcal{F}(t) \\
& +\sum_{j=1}^{N} \mathcal{G}_{j}^{T}(t) \sum_{i=1}^{N}\left(p_{1} Z_{1}^{i}+\left(p_{2}-p_{1}\right) Z_{2}^{i}\right) \mathcal{G}_{j}(t) \\
& -\sum_{i=1}^{N} \int_{t-p_{1}}^{t}\left(\mathcal{F}^{T}(s) Z_{1}^{i} \mathcal{F}(s)+\sum_{j=1}^{N} \mathcal{G}_{j}^{T}(s) Z_{1}^{i} \mathcal{G}_{j}(s)\right) d s \\
& \left.-\sum_{i=1}^{N} \int_{t-p_{2}}^{t-p_{1}}\left(\mathcal{F}^{T}(s) Z_{2}^{i} \mathcal{F}(s)+\sum_{j=1}^{N} \mathcal{G}_{j}^{T}(s) Z_{2}^{i} \mathcal{G}_{j}(s)\right) d s\right\} .
\end{aligned}
$$

It is not difficult to verify that

$$
\begin{aligned}
& -\mathbb{E}\left\{\sum_{i=1}^{N} \int_{t-p_{1}}^{t}\left(\mathcal{F}^{T}(s) Z_{1}^{i} \mathcal{F}(s)+\sum_{j=1}^{N} \mathcal{G}_{j}^{T}(s) Z_{1}^{i} \mathcal{G}_{j}(s)\right) d s\right\} \\
= & -\mathbb{E}\left\{\sum_{i=1}^{N} \int_{t-p_{1}}^{t} \dot{e}^{T}(s) Z_{1}^{i} \dot{e}(s) d s\right\}, \\
& -\mathbb{E}\left\{\sum_{i=1}^{N} \int_{t-p_{2}}^{t-p_{1}}\left(\mathcal{F}^{T}(s) Z_{2}^{i} \mathcal{F}(s)+\sum_{j=1}^{N} \mathcal{G}_{j}^{T}(s) Z_{2}^{i} \mathcal{G}_{j}(s)\right) d s\right\} \\
= & -\mathbb{E}\left\{\sum_{i=1}^{N} \int_{t-p_{2}}^{t-p_{1}} \dot{e}^{T}(s) Z_{2}^{i} \dot{e}(s) d s\right\} .
\end{aligned}
$$

Then, it follows from Lemma 2 that

$$
\begin{aligned}
- & \mathbb{E}\left\{\sum_{i=1}^{N} \int_{t-p_{1}}^{t} \dot{e}^{T}(s) Z_{1}^{i} \dot{e}(s) d s\right\} \\
\leq & \mathbb{E}\left\{-\frac{1}{p_{1}} \sum_{i=1}^{N}\left(e(t)-e\left(t-\tau_{1}^{i}(t)\right)\right)^{T} Z_{1}^{i}\left(e(t)-e\left(t-\tau_{1}^{i}(t)\right)\right)\right. \\
& -\frac{1}{p_{1}} \sum_{i=1}^{N}\left(e\left(t-\tau_{1}^{i}(t)\right)-e\left(t-p_{1}\right)\right)^{T} Z_{1}^{i} \\
& \left.\times\left(e\left(t-\tau_{1}^{i}(t)\right)-e\left(t-p_{1}\right)\right)\right\},
\end{aligned}
$$

and

$$
\begin{aligned}
& -\mathbb{E}\left\{\sum_{i=1}^{N} \int_{t-p_{2}}^{t-p_{1}} \dot{e}^{T}(s) Z_{2}^{i} \dot{e}(s) d s\right\} \\
\leq & \mathbb{E}\left\{-\frac{1}{p_{2}-p_{1}} \sum_{i=1}^{N}\left(e\left(t-p_{1}\right)-e\left(t-\tau_{2}^{i}(t)\right)\right)^{T} Z_{2}^{i}\right. \\
& \times\left(e\left(t-p_{1}\right)-e\left(t-\tau_{2}^{i}(t)\right)\right)-\frac{1}{p_{2}-p_{1}} \sum_{i=1}^{N}\left(e\left(t-\tau_{2}^{i}(t)\right)\right. \\
& \left.\left.-e\left(t-p_{2}\right)\right)^{T} Z_{2}^{i}\left(e\left(t-\tau_{2}^{i}(t)\right)-e\left(t-p_{2}\right)\right)\right\} .
\end{aligned}
$$

Substituting (19)-(22) into (18) yields

$\mathbb{E}\left\{\mathcal{L} V\left(e_{t}\right)\right\} \leq \mathbb{E}\left\{\xi^{T}(t) \bar{\Phi}_{1} \xi(t)+\mathcal{F}^{T}(t) R \mathcal{F}(t)+\sum_{j=1}^{N} \mathcal{G}_{j}^{T}(t) R \mathcal{G}_{j}(t)\right\}$ 
where

$$
\begin{aligned}
& \xi(t)=\left[\begin{array}{lllll}
e^{T}(t) & e_{\tau_{1}}^{T}(t) & e^{T}\left(t-p_{1}\right) & e_{\tau_{2}}^{T}(t) \\
& e^{T}\left(t-p_{2}\right) & g^{T}(x(t), s(t))
\end{array}\right]^{T}, \\
& e_{\tau_{1}}(t)=\left[\begin{array}{lllll}
e^{T}\left(t-\tau_{1}^{1}(t)\right) & \cdots & e^{T}\left(t-\tau_{1}^{N}(t)\right)
\end{array}\right]^{T}, \\
& e_{\tau_{2}}(t)=\left[\begin{array}{lcccc}
e^{T}\left(t-\tau_{2}^{1}(t)\right) & \cdots & e^{T}\left(t-\tau_{2}^{N}(t)\right)
\end{array}\right]^{T}, \\
& \bar{\Phi}_{1}= \\
& {\left[\begin{array}{cccccc}
\bar{\Theta}_{11} & \Theta_{12} & 0 & P C & 0 & P \\
* & -\frac{2}{p_{1}} \hat{Z}_{1} & \frac{1}{p_{1}} \tilde{Z}_{1}^{T} & 0 & 0 & 0 \\
* & * & \Theta_{33} & \frac{1}{p_{2}-p_{1}} \tilde{Z}_{2} & 0 & 0 \\
* & * & * & -\frac{2}{p_{2}-p_{1}} \hat{Z}_{2} & \frac{1}{p_{2}-p_{1}} \tilde{Z}_{2}^{T} & 0 \\
* & * & * & * & \Theta_{55} & 0 \\
* & * & * & * & * & 0
\end{array}\right],} \\
& \bar{\Theta}_{11}=P(W \otimes \Gamma)+(W \otimes \Gamma)^{T} P+Q_{1}-\frac{1}{p_{1}} \sum_{i=1}^{N} Z_{1}^{i} .
\end{aligned}
$$

Considering the inequality (9), we have

$$
\begin{aligned}
& \mathbb{E}\left\{\mathcal{L} V\left(e_{t}\right)\right\} \\
& \leq \mathbb{E}\left\{\xi^{T}(t) \bar{\Phi}_{1} \xi(t)+\mathcal{F}^{T}(t) R \mathcal{F}(t)+\sum_{j=1}^{N} \mathcal{G}_{j}^{T}(t) R \mathcal{G}_{j}(t)\right. \\
&\left.\quad-\lambda\left[\begin{array}{c}
e(t) \\
g(x(t), s(t))
\end{array}\right]^{T}\left[\begin{array}{cc}
\tilde{U}_{1 \Lambda} & \tilde{U}_{2 \Lambda} \\
* & I
\end{array}\right]\left[\begin{array}{c}
e(t) \\
g(x(t), s(t))
\end{array}\right]\right\} \\
&=\mathbb{E}\left\{\xi^{T}(t) \bar{\Phi}_{2} \xi(t)+\mathcal{F}^{T}(t) R \mathcal{F}(t)+\sum_{j=1}^{N} \mathcal{G}_{j}^{T}(t) R \mathcal{G}_{j}(t)\right\}
\end{aligned}
$$

where

$$
\bar{\Phi}_{2}=\left[\begin{array}{cccccc}
\Theta_{11} & \Theta_{12} & 0 & P \vec{C} & 0 & \Theta_{16} \\
* & -\frac{2}{p_{1}} \hat{Z}_{1} & \frac{1}{p_{1}} \tilde{Z}_{1}^{T} & 0 & 0 & 0 \\
* & * & \Theta_{33} & \frac{1}{p_{2}-p_{1}} \tilde{Z}_{2} & 0 & 0 \\
* & * & * & -\frac{2}{p_{2}-p_{1}} \hat{Z}_{2} & \frac{1}{p_{2}-p_{1}} \tilde{Z}_{2}^{T} & 0 \\
* & * & * & * & \Theta_{55} & 0 \\
* & * & * & * & * & -\lambda I
\end{array}\right]
$$

By using the Schur complement [4], we obtain immediately from (16) that $\mathbb{E}\left\{\mathcal{L} V\left(e_{t}\right)\right\}<0$ which implies that there exists a sufficiently small constant $\varepsilon>0$ such that $\mathbb{E}\left\{\mathcal{L} V\left(e_{t}\right)\right\}<$ $-\varepsilon \mathbb{E}\left\{\|e(t)\|^{2}\right\}$. To this end, it follows immediately from Lemma 1 that the exponential mean-square stability of the augmented system (8) is guaranteed and, therefore, the proof of this theorem is complete.

It is worth mentioning that, in the proof of Theorem 1, the Jenson integral inequality is employed to derive a stability criterion for the dynamic system (8) with MPIDs and SBNs. The usage of the Jenson integral inequality is mainly to simplify the proof without causing much conservatism. In what follows, we shall deal with the design problem of controllers that make the dynamical network (1) exponentially mean-square synchronized for all probabilistic sampling periods. According to the stability criterion given in Theorem 1 , the following theorem is easily accessible.

Theorem 2: The dynamical network (1) is exponentially mean-square synchronized by controllers of the form (4) for all probabilistic sampling periods if there exist matrices $P=$ $\operatorname{diag}_{N}\{\bar{P}\}>0, X, Q_{1}>0, Q_{2}>0, Z_{1}^{i}>0, Z_{2}^{i}>0(1 \leq i \leq N)$, and a scalar $\lambda>0$ satisfying

$$
\left[\begin{array}{cc}
\Theta_{11} & \bar{\Theta}_{12} \\
* & -\frac{2}{p_{1}} \hat{Z}_{1} \\
* & * \\
* & * \\
* & * \\
* & * \\
* & * \\
* & *
\end{array}\right.
$$

$$
\begin{gathered}
0 \\
\frac{1}{p_{1}} \tilde{Z}_{1}^{T} \\
\Theta_{33} \\
* \\
* \\
* \\
* \\
*
\end{gathered}
$$$$
\begin{array}{cc}
\vec{Y} & 0 \\
0 & 0 \\
\frac{1}{p_{2}-p_{1}} \tilde{Z}_{2} & 0 \\
-\frac{2}{p_{2}-p_{1}} \hat{Z}_{2} & \frac{1}{p_{2}-p_{1}} \tilde{Z}_{2}^{T} \\
* & \Theta_{55} \\
* & * \\
* & * \\
* & *
\end{array}
$$$$
\left.\begin{array}{ccc}
\Theta_{16} & \left(W^{T} \otimes \Gamma^{T}\right) P & 0 \\
0 & \tilde{Y}^{T} & \hat{Y}^{T} \\
0 & 0 & 0 \\
0 & \vec{Y}^{T} & -\hat{Y}^{T} \\
0 & 0 & 0 \\
-\lambda I & P & 0 \\
* & -2 P+R & 0 \\
* & * & -2 \hat{P}+\hat{R}
\end{array}\right]<0
$$

where

$$
\begin{aligned}
& \bar{\Theta}_{12}=\tilde{Y}+\frac{1}{p_{1}} \tilde{Z}_{1}, \quad \tilde{Y}=\left[\begin{array}{lll}
\alpha_{1} Y_{1} & \cdots & \alpha_{N} Y_{N}
\end{array}\right], \\
& \vec{Y}=\left[\begin{array}{lll}
\left(1-\alpha_{1}\right) Y_{1} & \cdots & \left(1-\alpha_{N}\right) Y_{N}
\end{array}\right], \\
& \hat{Y}=\operatorname{diag}\left\{\sqrt{\alpha_{1}\left(1-\alpha_{1}\right)} Y_{1}, \cdots, \sqrt{\alpha_{N}\left(1-\alpha_{N}\right)} Y_{N}\right\} \text {, } \\
& Y_{i}=\operatorname{diag}\{\underbrace{0, \cdots, 0}_{i-1}, X, 0, \cdots, 0\}, \quad \hat{P}=\operatorname{diag}_{N}\{P\} \text {, }
\end{aligned}
$$

and $R, \hat{R}, \hat{Z}_{1}, \tilde{Z}_{1}, \hat{Z}_{2}, \tilde{Z}_{2}, \Theta_{11}, \Theta_{16}, \Theta_{33}$, and $\Theta_{55}$ are defined in Theorem 1. Furthermore, if the linear matrix inequality (LMI) (24) is solvable, the desired controller gain is given as $K=$ $\bar{P}^{-1} X$.

Remark 3: First, it can be seen from (24) that, if one of the sampling periods $p_{1}$ and $p_{2}\left(p_{1}<p_{2}\right)$ approaches infinity, the LMI problem would have no feasible solution. In most cases, the sampling periods $p_{1}$ and $p_{2}$ take deterministic values, and this is helpful for the solvability of the LMI problem. Second, due to the existence of the coupled term $\sum_{j=1}^{N} w_{i j} \Gamma e_{j}(t)$, the conditions in Theorems 1 and 2 are more feasible when the coupling strength $w_{i j}$ is getting larger. Moreover, the parameters $U_{1}$ and $U_{2}$, which are used to characterize the nonlinearities in the network, also affect the conditions in Theorem 2. The less is $U_{2}-U_{1}$, the less is $\mathbb{E}\left\{\mathcal{L} V\left(e_{t}\right)\right\}$, which is better for the system (8) to maintain its stability.

\section{An Illustrative Example}

Consider a dynamical network (1) with three nodes. The coupling configuration matrix $W=\left(w_{i j}\right)_{3 \times 3}$ is given by $w_{i j}=$ $-2, i=j, w_{i j}=1, i \neq j$, and the inner-coupling matrix is set as $\Gamma=I$. The nonlinear function in the dynamical network (1) is chosen as

$$
f\left(x_{i}(t)\right)=\left[\begin{array}{c}
-0.5 x_{i 1}(t)+\tanh \left(0.2 x_{i 1}(t)\right)+0.2 x_{i 2}(t) \\
0.65 x_{i 2}(t)-\tanh \left(0.45 x_{i 2}(t)\right)
\end{array}\right] .
$$

It is easy to verify that above nonlinear function $f(\cdot)$ satisfies the sector-bounded condition (2) with

$$
U_{1}=\left[\begin{array}{cc}
-0.5 & 0.2 \\
0 & 0.65
\end{array}\right], \quad U_{2}=\left[\begin{array}{cc}
-0.3 & 0.2 \\
0 & 0.2
\end{array}\right]
$$

In this example, the sampling periods are taken as $p_{1}=0.02$ and $p_{2}=0.04$, and the probabilities are set as $\beta_{1}=0.75, \beta_{2}=$ 

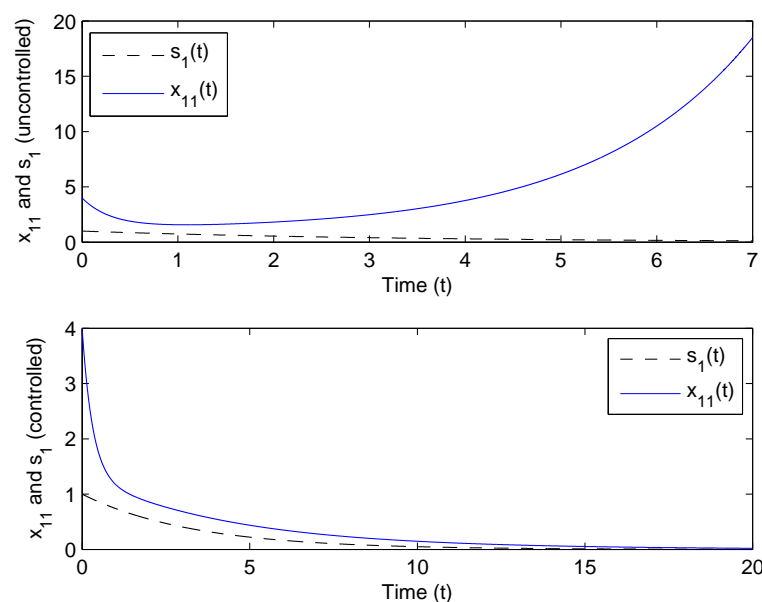

Fig. 1. State $x_{11}$ of uncontrolled and controlled node 1
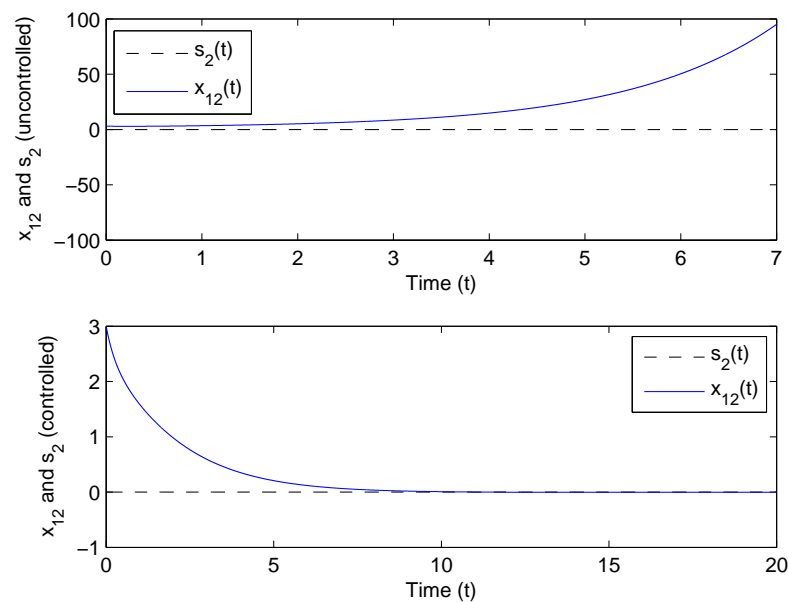

Fig. 2. State $x_{12}$ of uncontrolled and controlled node 1

0.8 , and $\beta_{3}=0.7$. By using the Matlab (with YALMIP 3.0 and SeDuMi 1.1), we solve LMI (24) and obtain the parameter of the desired controllers as follows:

$$
K=\bar{P}^{-1} X=\left[\begin{array}{cc}
0.1312 & -0.1699 \\
-0.0411 & -0.6978
\end{array}\right] \text {. }
$$

According to Theorem 2, the set of controllers (4) with the parameter given by (25) can achieve the exponential mean-square synchronization of the considered dynamical network. Simulation results are shown in Figs. 1-2, from which it can be observed that 1 ) the state trajectories of all the three nodes (we only list the plots for the first node to save space) deviate from one of the isolate node drastically in the case that there is no controller to the dynamical network; and 2) all state trajectories of the controlled network can converge to the one governed by the identical isolate node, which confirm our main results.

\section{Conclusions}

In this paper, the sampled-data synchronization control problem has been addressed for a class of dynamical networks with stochastic sampling. The addressed synchronization control problem has first been transformed to the exponential meansquare stability analysis problem for a dynamic system with
MPIDs as well as SBNs. By constructing a new Lyapunov functional and employing Gronwall's inequality and Jenson integral inequality, a sufficient condition has been obtained to guarantee the exponential mean-square stability of the considered dynamic system and the set of sampled-data synchronization controllers has been designed. In our future work, we will further consider the problems for randomly occurring nonlinearities and randomly occurring network topology, which would reflect more features of the complexity.

\section{REFERENCES}

[1] R. Albert and A. L. Barabási, Statistical mechanics of complex networks, Rev. Modern Phys., Vol. 74, No. 1, pp. 47-97, Jan. 2002.

[2] B. Bamieh and J. Pearson, A general framework for linear periodic-systems with applications to $H_{\infty}$ sampled-data control, IEEE Trans. Automatic Control, Vol. 37, No. 4, pp. 418-435, Apr. 1992.

[3] M. Basin, P. Shi and D. Calderon-Alvarez, Central suboptimal $H_{\infty}$ filter design for linear time-varying systems with state and measurement delays, Int. J. Systems Science, Vol. 41, No. 4, pp. 411-421, 2010.

[4] S. Boyd, L. E. Ghaoui, E. Feron and V. Balakrishnan, Linear Matrix Inequalities in System and Control Theory, Philadelphia: SIAM, 1994.

[5] T. Chen and B. Francis, Optimal Sampled-Data Control Systems, Springer: London, 1995

[6] T. Chen, X. Liu and W. Lu, Pinning complex networks by a single controller, IEEE Trans. Circuits and Systems -I: Regular Papers, Vol. 54, No. 6, pp. 1317-1326, Jun. 2007.

[7] R. L. Cook, Stochastic sampling in computer graphics, ACM Trans. Graph., Vol. 5, No. 1, pp. 51-72, Jan. 1986.

[8] E. Fridman, U. Shaked and V. Suplin, Input/output delay approach to robust sampled-data $H_{\infty}$ control, Systems $\&$ Control Letters, Vol. 54, No. 3 , pp. 271-282, Mar. 2005.

[9] H. Gao, J. Wu and P. Shi, Robust sampled-data $H_{\infty}$ control with stochastic sampling, Automatica, Vol. 45, No. 7, pp. 1729-1736, Jul. 2009.

[10] D. P. Goodall and R. Postoyan, Output feedback stabilisation for uncertain nonlinear time-delay systems subject to input constraints, Int. J. Control, Vol. 83, No. 4, pp. 676-693, 2010.

[11] K. Gu, An integral inequality in the stability problem of time-delay systems, Proc. 39th IEEE Conf. Decision and Control, Sydney, Australia, pp. 2805-2810, Dec. 2000.

[12] H. Karimi and H. Gao, LMI-based $H_{\infty}$ synchronization of second-order neutral master-slave systems using delayed output feedback control, International Journal of Control, Automation and Systems, Vol. 7, No. 3, pp. 371380, Jun. 2009.

[13] H. Karimi and H. Gao, New delay-dependent exponential $H_{\infty}$ synchronization for uncertain neural networks with mixed time delays, IEEE Trans. Systems, Man and Cybernetics-Part B, Vol. 40, No. 1, pp. 173-185, 2010.

[14] H. Karimi and P. Maass, Delay-range-dependent exponential $H_{\infty}$ synchronization of a class of delayed neural networks, Chaos, Solitons $\&$ Fractals, Vol. 41, No. 3, pp. 1125-1135, Aug. 2009.

[15] P. Li, J. Lam and Z. Shu, On the transient and steady-State estimates of interval genetic regulatory networks, IEEE Trans. Systems, Man and Cybernetics-Part B, Vol. 40, No. 2, pp. 336-349, 2010.

[16] Y. Liu, Z. Wang, J. Liang and X. Liu, Synchronization and state estimation for discrete-time complex networks with distributed delays, IEEE Trans. Systems, Man and Cybernetics-Part B, Vol. 38, No. 5, pp. 1314-1325, Oct. 2008.

[17] J. Lü and G. Chen, A time-varying complex dynamical network model and its controlled synchronization criteria, IEEE Trans. Automatic Control, Vol. 50, No. 6, pp. 841-846, Jun. 2005.

[18] W. Lu, X. Li and Z. Rong, Global stabilization of complex networks with digraph topologies via a local pinning algorithm, Automatica, Vol. 46, No. 1, pp. 116-121, 2010.

[19] X. Mao, Stochastic Differential Equations and Their Applications, Chichester: Horwood Publisher, 1997.

[20] H. Su, X. Wang and Z. Lin, Synchronization of coupled harmonic oscillators in a dynamic proximity network, Automatica, Vol. 45, No. 10, pp. 22862291, 2009.

[21] S. H. Strogatz, Exploring complex networks, Nature, Vol. 410, No. 6825, pp. 268-276, Mar. 2001.

[22] Y. Yamamoto, New approach to sampled-data control systems-a function space method, Proceedings of IEEE conference on decision and control, Honolulu, HW, pp. 1882-1887, 1990.

[23] W. Yu, G. Chen and J. Lü, On pinning synchronization of complex dynamical networks, Automatica, Vol. 45, No. 2, pp. 429-435, 2009.

[24] D. Yue, E. Tian, Z. Wang and J. Lam, Stabilization of systems with probabilistic interval input delays and its applications to networked control systems, IEEE Trans. Systems, Man and Cybernetics-Part A, Vol. 39, No. 4, pp. 939-945, Jul. 2009.

[25] J. Zhou and T. Chen, Synchronization in general complex delayed dynamical networks, IEEE Trans. Circuits and Systems -I: Regular Papers, Vol. 53, No. 3, pp. 733-744, Mar. 2006. 